\title{
Short communication: Estimation of genetic parameters for residual feed intake and feeding behavior traits in dairy heifers
}

\author{
Z. Lin, ${ }^{* 1}$ I. Macleod, ${ }^{*}+$ and J. E. Pryce†‡ \\ ${ }^{*}$ Faculty of Land and Food Resources, University of Melbourne, Parkville 3010, Australia \\ †Biosciences Research Division, Department of Primary Industries Victoria, 5 Ring Road, Bundoora 3083, Australia \\ $\ddagger$ Dairy Futures Co-operative Research Centre, 1 Park Drive, Bundoora 3083, Australia
}

\begin{abstract}
Data from a 2-yr feeding trial of Holstein-Friesian heifers $(\mathrm{n}=842)$ were used to examine the heritability of feeding behavior traits and their relationships with residual feed intake (RFI), a measure of feed efficiency. Five traits were assessed: number of meals, feeding duration, dry matter intake (DMI), eating rate, and average meal size. For estimating genetic parameters, all traits were simultaneously fitted in a multivariate model with a genomic relationship matrix calculated from heifers' high-density genotype data. All 5 traits were moderately heritable (0.45-0.50), which was slightly higher than the estimate for RFI $(0.40 \pm 0.09)$. Two traits had modest genetic correlations with RFI (DMI and feeding duration; $0.45 \pm 0.13$ and $0.27 \pm$ 0.15 , respectively), and 2 traits had modest phenotypic correlations with RFI (DMI and eating rate; $0.52 \pm 0.03$ and $0.23 \pm 0.04$, respectively). The results indicate that feeding behavior (1) may differ between efficient and inefficient animals and (2) may be useful for selecting animals with better feed efficiency. However, the limitation is that measurements on DMI are still essential. It is therefore possible that a more efficient selection tool for RFI may be the use of high-density DNA markers to make direct genomic predictions for RFI.
\end{abstract}

Key words: correlation, feeding behavior, residual feed intake, heifer

\section{Short Communication}

Feed efficiency is an important trait that has a major influence on production costs in the dairy industry. Although residual feed intake (RFI), defined as the difference between actual and predicted feed intake (Koch et al., 1963), is gaining popularity as a measure of feed efficiency, it is both expensive and time-consuming to measure. Thus, it is of interest to study alternative

Received September 6, 2012.

Accepted December 4, 2012.

${ }^{1}$ Corresponding author: linzibei610@hotmail.com traits, such as feeding behavior, to determine if these may indirectly predict feed efficiency.

In human and rodent studies, evidence exists relating meal frequency, meal size (MS), and eating speed to different metabolic rates and obesity (Heinrichs, 2001; Scisco et al., 2011). Recent studies also suggest modest phenotypic and genetic correlations in beef cattle between RFI, meal frequency (Kelly et al., 2010), and feeding duration (FD; Durunna et al., 2011); however, these estimates are very imprecise. Currently, no published estimates of these correlations for dairy cattle exist.

The main objectives of this study were to estimate genetic and phenotypic parameters for RFI, and feeding behavior traits in growing dairy heifers. We hypothesized that the results would be useful in determining which of these traits influence feed efficiency in young dairy heifers, and whether feeding behavior could be a useful alternative to select for more efficient dairy cattle.

A total of 842 Holstein-Friesian heifers aged around 6 mo from 23 farms in northern Victoria, Australia, were used in this experiment. The experiment was run in 3 batches over a 2-yr period, with around 300 heifers in each batch. Animals were grouped according to their farm of origin at the test facility and were all fed the same cubed alfalfa feed. The feeders were designed specifically for the experiment by Gallagher Animal Management Systems, Hamilton, New Zealand. A complete description of the design of this experiment can be found in the study of Williams et al. (2011).

Feed intake data were collected over a continuous 56 -d test period in each batch, and animal BW were checked weekly over the same period. Four feeding behavior traits were calculated from feed intake data measured using automated feeders that recorded every meal event. Although DMI is not strictly a feeding behavior trait, it was included as an additional trait in this study. The traits were: number of meal events (NM; no./d); FD (h/d); DMI (kg/d); eating rate (ER; $\mathrm{kg} / \mathrm{h})$, calculated as DMI/FD; and MS ( $\mathrm{kg} /$ meal), calculated as DMI/NM. All traits were calculated from automated data recorded over each 24-h day and then 
Table 1. Mean $( \pm \mathrm{SD})$ for feeding traits ${ }^{1}$ throughout the experiment

\begin{tabular}{lcccc}
\hline Item & Batch 1 & Batch 2 & Batch 3 & Total \\
\hline Animals (no./d) & 278 & 265 & 299 & 842 \\
RFI (kg/d) & $0.0 \pm 0.47$ & $0.0 \pm 0.40$ & $0.0 \pm 0.42$ & $0.0 \pm 0.42$ \\
NM (no./d) & $11.8 \pm 3.6$ & $11.7 \pm 7.0$ & $15.4 \pm 8.0$ & $13.5 \pm 1.3$ \\
FD (h/d) & $2.3 \pm 0.3$ & $2.6 \pm 0.4$ & $2.5 \pm 0.4$ & $2.4 \pm 0.3$ \\
DMI (kg/d) & $8.8 \pm 1.2$ & $7.1 \pm 1.2$ & $8.4 \pm 1.2$ & $8.2 \pm 0.6$ \\
ER (kg/h) & $4.4 \pm 0.9$ & $3.3 \pm 0.8$ & $4.0 \pm 0.9$ & $3.9 \pm 0.8$ \\
MS (kg/meal) & $0.8 \pm 0.2$ & $0.8 \pm 0.3$ & $0.6 \pm 0.2$ & $0.7 \pm 0.1$ \\
\hline${ }^{1}$ RFI = residual feed intake; NM = number of meals; FD = feeding duration; ER = eating rate; MS = meal size
\end{tabular}

averaged over the entire test period for each of the animals. The ER and MS were based on DMI. The RFI was calculated for each cohort of heifers separately:

$$
\begin{aligned}
\mathrm{RFI} & =\mathrm{DMI}-(\mu+\mathrm{BW}+\mathrm{BW} \Delta \\
& \left.+ \text { farm }+ \text { age }+\mathrm{age}^{2}\right),
\end{aligned}
$$

where $\mu$ is the overall mean effect; $\mathrm{BW}$ is the testperiod midpoint $\mathrm{BW}$, and $\mathrm{BW} \Delta$ is growth rate; farm is the animal group of origin; age is animals' age at the start of test. More details of the RFI estimation can be found in the study of Pryce et al. (2012).

Genetic parameters for the feeding behavior traits and RFI were estimated in this study using linear mixed models in ASreml (Gilmour et al., 2006). The model is shown as follows:

$$
\begin{aligned}
\mathrm{y}_{\mathrm{ijk}}=\mu & + \text { farm }_{\mathrm{i}}+\text { batch }_{\mathrm{j}}+\text { pen }_{\mathrm{k}}+\mathrm{b}_{1} \text { age } \\
& +\mathrm{b}_{2} \mathrm{BW}+\text { animal }+\mathrm{e}_{\mathrm{ijk}},
\end{aligned}
$$

where $\mu$ is the overall phenotypic mean of each trait and e is the error term; farm ${ }_{\mathrm{i}}$, batch $_{\mathrm{j}}$, and pen $_{\mathrm{k}}$ are fixed effects; age at the start of the test and BW at the midpoint of the test period are fitted as covariates, and $b_{1}$ and $b_{2}$ are the respective regressions coefficients on these traits; animal is a random effect to estimate the additive genetic variation $\left(\sigma_{G}^{2}\right)$, which is modeled as $\mathbf{A} \sigma_{G}^{2}$. Here, 2 alternative $\mathbf{A}$ matrices were used: one from pedigree relationships, and the other a genomic relationship matrix (GRM) calculated using the heifers' genotype data. All heifers were genotyped by using the Illumina High Density Bovine SNP chip, which comprises 777,963 SNP markers (Illumina Inc., San Diego, CA) of which 624,930 passed quality control criteria. A full description of the genotype data, the quality control process, and construction of the GRM can be found in the study of Pryce et al. (2012).

Initially, univariate and bivariate models were tested for each target trait to check the statistical significance of fixed effects and to obtain starting values for the (co) variance components. A multivariate model was then run with all 6 traits, fitting only the significant fixed effects $(P<0.01)$ for each trait.

A summary of the uncorrected means within and across batches for the 5 feeding traits (mean \pm SD) are shown in Table 1. The standard deviations were consistent across all 3 batches.

Heritability estimates are shown in Table 2. As mentioned earlier, 2 genetic matrices were used in the model (pedigree and GRM); however, convergence problems were encountered with the pedigree matrix. Thus, only the results estimated using the multivariate GRM model are shown here. All feeding behavior traits were found to be moderately heritable and generally in agreement with those found in beef cattle (Robinson and Oddy, 2004; Durunna et al., 2011).

The genetic correlations between traits are shown in Table 2. Here, the moderate genetic correlation between RFI and DMI $(0.45 \pm 0.13)$ is in agreement with several published studies, for example, 0.43 in Robinson and Oddy (2004) and 0.85 in Lancaster et al. (2009). The genetic correlation between RFI and FD ( $0.27 \pm$ 0.15) was also consistent with the results of Robinson and Oddy (2004) and Nkrumah et al. (2007; $0.35 \pm$

Table 2. Heritability estimates (on the diagonal) and genetic and phenotypic correlations (above and below the diagonal, respectively) for feeding traits ${ }^{1}$

\begin{tabular}{lccccrr}
\hline Item & \multicolumn{1}{c}{ RFI } & NM & FD & DMI & \multicolumn{1}{c}{ ER } & \multicolumn{1}{c}{ MS } \\
\hline RFI & $\mathbf{0 . 4 0 ( 0 . 0 9 )}$ & $-0.07(0.17)$ & $0.27(0.15)$ & $0.45(0.13)$ & $0.06(0.16)$ & $-0.06(0.16)$ \\
NM & $0.05(0.04)$ & $\mathbf{0 . 4 5 ( 0 . 0 8 )}$ & $0.03(0.14)$ & $0.03(0.16)$ & $-0.01(0.15)$ & $-0.80(0.06)$ \\
FD & $0.11(0.04)$ & $-0.04(0.04)$ & $\mathbf{0 . 5 0 ( 0 . 0 9 )}$ & $0.48(0.12)$ & $-0.81(0.04)$ & $-0.23(0.14)$ \\
DMI & $0.52(0.03)$ & $0.09(0.04)$ & $0.32(0.04)$ & $\mathbf{0 . 4 6 ( 0 . 0 9 )}$ & $0.11(0.14)$ & $0.18(0.15)$ \\
ER & $0.23(0.04)$ & $0.10(0.04)$ & $-0.80(0.01)$ & $0.23(0.04)$ & $\mathbf{0 . 4 6 ( 0 . 0 9 )}$ & $-0.13(0.14)$ \\
MS & $0.06(0.04)$ & $-0.76(0.02)$ & $0.10(0.04)$ & $0.13(0.04)$ & $-0.04(0.04)$ & $\mathbf{0 . 4 8 ( 0 . 0 9 )}$ \\
\hline
\end{tabular}

${ }^{1} \mathrm{RFI}=$ residual feed intake; $\mathrm{NM}=$ number of meals; $\mathrm{FD}=$ feeding duration; $\mathrm{ER}=$ eating rate; $\mathrm{MS}=$ meal size 
0.17 and $0.57 \pm 0.28$, respectively). For the genetic correlation between RFI and the other 3 traits (NM, ER, and MS), none were significantly different from zero. In other published studies, potential genetic correlations were estimated between RFI and NM $(0.34 \pm 0.30$; Nkrumah et al., 2007), as well as between RFI and ER $(0.18 \pm 0.31$; Durunna et al., 2011). However, it should be noted that the standard errors are as large as or larger than the estimates, and no genetic correlations between RFI and these traits may actually exist.

The phenotypic correlations between traits are also shown in Table 2. A moderate to strong positive phenotypic correlation between RFI and DMI $(0.52 \pm 0.03)$ is in agreement with other studies (Robinson and Oddy (2004); Kelly et al., 2010). This implies that high-intake animals either have a higher rate of food passage with relatively less time for complete digestion and nutrient uptake, or they use energy less efficiently for growth compared with those with lower intakes. It would be useful to understand if this is due to biological inefficiencies of the animal, or if high intakes and relatively larger meal size could hinder rumen fermentation. However, the weak phenotypic correlation between RFI and MS (0.06) implies that meal size has no effect on RFI. Residual feed intake and ER had a moderate phenotypic correlation in our study (0.23), whereas it was not significantly different from zero in Durunna et al. (2011). The biological differences of sex or growth stage may be a possible explanation, with growing heifers in our study and finished steers in theirs.

Interestingly, FD was found to have moderate positive correlations with DMI and strong negative correlations with ER (genetic and phenotypic); only a weak positive correlation between ER and DMI exists (Table 2). This implies that slow eaters are generally spending more time eating, thus their total DMI is hardly affected by their eating rate. Meal size was found to have strong negative genetic and phenotypic correlations with NM, whereas it only had a weak correlation with DMI. This indicates that, generally, animals with a preference for small meals also tend to have more meal events to satisfy their DMI needs. Therefore, neither ER nor MS was a good indicator of DMI. In future studies it may be interesting to look at correlations between RFI and more detailed feeding behavior traits, such as DMI at different times of day.

In conclusion, some traits have moderate or strong genetic and phenotypic correlations with RFI (i.e., DMI, FD, and ER). Dry matter intake is used in the calculation of RFI and, therefore, is not a feeding behavior trait, whereas FD is an indirect measure of DMI. Heifers that eat for longer are obviously expected to eat more; therefore, we concluded although feeding behavior may help to achieve our goal of indirectly selecting more efficient cattle - the limitation is that a measure of feed intake is still required. Additionally, ER was phenotypically correlated to RFI and DMI. This is of interest because it implies that cattle that eat slowly have improved feed efficiency, although it is not effective to use ER as a selection criterion to genetically improve RFI. Thus, we suggest that genomic selection tools for the trait of interest (i.e., RFI) may be better than indirect selection criteria (Pryce et al., 2012).

\section{ACKNOWLEDGMENTS}

The authors thank the staff involved with the trial, especially Marg Jenkin, Gareth "Taffy" Phillips, Mel Porker, Ben Hayes, Bill Wales, and Yvette Williams. We also thank the owners of the heifers used in this trial. We gratefully acknowledge the Geoffrey Gardiner Dairy Foundation (Melbourne, Australia), Dairy Futures Co-operative Research Council (Melbourne, Australia), and Department of Primary Industries (Melbourne, Australia) for funding the research.

\section{REFERENCES}

Durunna, O. N., Z. Wang, J. A. Basarab, E. K. Okine, and S. S Moore. 2011. Phenotypic and genetic relationships among feeding behavior traits, feed intake, and residual feed intake in steers fed grower and finisher diets. J. Anim. Sci. 89:3401-3409.

Gilmour, A. R., B. J. Gogel, B. R. Cullis, and R. Thompson. 2006. ASReml User Guide Release 2.0. VSN International Ltd., Hemel Hempstead, UK.

Heinrichs, S. C. 2001. Mouse feeding behavior: Ethology, regulatory mechanisms and utility for mutant phenotyping. Behav. Brain Res. 125:81-88.

Kelly, A. K., M. McGee, D. H. Crews, A. G. Fahey, A. R. Wylie, and D. A. Kenny. 2010. Effect of divergence in residual feed intake on feeding behavior, blood metabolic variables, and body composition traits in growing beef heifers. J. Anim. Sci. 88:109-123.

Koch, R. M., L. A. Swiger, D. Chambers, and K. E. Gregory. 1963. Efficiency in beef cattle. J. Anim. Sci. 22:486-494.

Lancaster, P. A., G. E. Carstens, D. H. Crews, T. H. Welsh, T. D A. Forbes, D. W. Forrest, L. O. Tedeschi, R. D. Randel, and F. M. Rouquette. 2009. Phenotypic and genetic relationships of residual feed intake with performance and ultrasound carcass traits in Brangus heifers. J. Anim. Sci. 87:3887-3896.

Nkrumah, J. D., D. H. Crews, J. A. Basarab, M. A. Price, E. K. Okine, Z. Wang, C. Li, and S. S. Moore. 2007. Genetic and phenotypic relationships of feeding behavior and temperament with performance, feed efficiency, ultrasound, and carcass merit of beef cattle. J. Anim. Sci. 85:2382-2390.

Pryce, J. E., J. Arias, P. J. Bowman, S. R. Davis, K. A. Macdonald, G. C. Waghorn, W. J. Wales, Y. J. Williams, R. J. Spelman, and B. J. Hayes. 2012. Accuracy of genomic predictions of residual feed intake and 250-day body weight in growing heifers using 625,000 single nucleotide polymorphism markers. J. Dairy Sci. 95:2108-2119.

Robinson, D. L., and V. H. Oddy. 2004. Genetic parameters for feed efficiency, fatness, muscle area and feeding behavior of feedlot finished beef cattle. Livest. Prod. Sci. 90:255-270.

Scisco, J. L., E. R. Muth, Y. J. Dong, and A. W. Hoover. 2011. Slowing bite-rate reduces energy intake: An application of the bite counter device. J. Am. Diet. Assoc. 111:1231-1235.

Williams, Y. J., J. E. Pryce, C. Grainger, W. J. Wales, N. Linden, M. Porker, and B. J. Hayes. 2011. Variation in residual feed intake in Holstein-Friesian dairy heifers in southern Australia. J. Dairy Sci. 94:4715-4725. 